\title{
Densidade e tamanho populacional de primatas em um fragmento florestal no sudeste do Brasil
}

\author{
Christine Steiner São Bernardo \& Mauro Galetti
}

Laboratório de Biologia da Conservação, Departamento de Ecologia, Universidade Estadual Paulista. Avenida 24-A 1515, Bela Vista, 13506-900 Rio Claro, São Paulo, Brasil.E-mail: christinesteiner@yahoo.com; mgaletti@rc.unesp.br

\begin{abstract}
Density and population size of primates in a forest fragment in southeastern Brazil. Linetransect surveys were conducted at a small remnant of semideciduous Atlantic Forest to estimate primate density and population size. The Mata São José has about 230 ha and it is located between Rio Claro and Araras (São Paulo State, southeastern Brazil). This forest fragment holds 23 non-volant mammal species distributed in seven orders e 13 families. From July 1999 to January 2001, a total of $125 \mathrm{~km}$ were walked and three primate species were recorded. The densities were estimated based on Distance software. Brown capuchin monkey Cebus nigritus (Goldfuss, 1809) (Cebidae) presented the highest population density (24.5 individuals/ km², ranging between 19.6 and 32.6 individuals $/ \mathrm{km}^{2}$ ). Titi monkeys Callicebus nigrifrons (Spix, 1823) (Pitheciidae) and tufted ear marmosets Callithrix aurita (É. Geoffroy, 1812) (Callitrichidae) presented 3.5 individuals/ km² (ranging between 2.8 to 4.6 individuals $/ \mathrm{km}^{2}$ ). Total population size of brown capuchin monkeys was 56 individuals (ranging between 45 to 75 individuals) and the titi monkeys and tufted ear marmosets were eight individuals (ranging between six to 10 individuals). Titi monkeys and tufted ear marmosets are vulnerable to extinction and both persist at Mata São José, but at low population densities. These primate species are at local extinction risk, because populations at low densities are more suscetible to stochastic events, inbreeding depression and environmental variation. Translocation is the only plausible management action that could avoid local extinction of these primates. KEY WORDS. Atlantic forest, census, forest fragmentation, line-transect method, primates.
\end{abstract}

RESUMO. Foram realizadas transecções lineares em um pequeno fragmento de Floresta Atlântica semidecídua, a fim de se estimar densidade e tamanho populacional de primatas. A Mata São José possui uma área de 230 ha e está localizada entre Rio Claro e Araras (Estado de São Paulo, sudeste do Brasil). Este fragmento florestal abriga 23 espécies de mamíferos não - voadores, distribuídos em sete ordens e 13 famílias. De julho de 1999 a janeiro de 2001, foram percorridos $125 \mathrm{~km}$ e três espécies de primatas foram registradas. As densidades populacionais foram estimadas com base no software Distance. A espécie de primata que apresentou maior densidade foi o macaco-prego Cebus nigritus (Goldfuss, 1809) (Cebidae) (24,5 indivíduos/ km², variando entre 19,6 e 32,6 indivíduos $/ \mathrm{km}^{2}$ ). Para o sauá Callicebus nigrifrons (Spix, 1823) (Pitheciidae) e o sagüi-da-serra-escuro Callithrix aurita (É. Geoffroy, 1812) (Callitrichidae) foi calculada uma densidade de 3,5 indivíduos/ $\mathrm{km}^{2}$ (variando entre 2,8 e 4,6 indivíduos $/ \mathrm{km}^{2}$ ). A população total de macaco - prego estimada foi de 56 indivíduos (variando de 45 a 75 indivíduos), bem como a população total de sauá e sagüii-da-serra-escuro foi de oito indivíduos (variando de seis a 10 indivíduos). O sauá e o sagüi-da-serra-escuro estão inclusos na lista nacional das espécies da fauna brasileira ameaçadas de extinção no Brasil, considerados vulneráveis à extinção, mas esses dois primatas ainda persistem na Mata São José, ainda que em baixa densidade populacional. Estas espécies estão correndo risco de extinção local, pois o pequeno tamanho das populações as torna mais suscetíveis a efeitos estocásticos, depressão genética por endocruzamento e variações ambientais. A translocação destas espécies de primatas é a única medida de manejo plausível que pode evitar a extinção local destas populações de primatas.

PALAVRAS CHAVE. Censo, floresta atlântica, fragmentação florestal, método de transecção linear, primatas.

O Estado de São Paulo apresenta atualmente poucos fragmentos de Floresta Atlântica, sendo que os maiores remanescentes contínuos do Estado situam-se na região do Vale do Ribeira, na região do Pontal do Paranapanema e ao longo da faixa litorânea (Sos Mata AtLÂNTica \& InPe 1998, Sma 2000). No interior paulista, as áreas florestais são pequenas e isoladas em sua gran- 
de maioria por pastagens, canaviais, plantações de café e cítricos (Dean 1996, Sos Mata Atlântica \& Inpe 1998). A Mata Atlântica nessa região é do tipo tropical semidecídua e foi um dos primeiros habitats a ser destruído durante a colonização, em função do clima e do solo ser mais adequado para a agricultura e para a pecuária (DeAn 1996, Sos Mata AtLÂNTICA \& INPE 1998). Esses arquipélagos de fragmentos possuem uma importante função na manutenção da biodiversidade local, porém vêm sofrendo forte pressão antrópica (como caça, fogo e corte seletivo) e aumento do efeito de borda (BierRegaARd et al. 1992, Turner \& CORlett 1996).

Diversos estudos em áreas florestais fragmentadas na Amazônia, e mais recentemente na Mata Atlântica, relacionaram a abundância, densidade, ocorrência e riqueza das espécies com o tamanho do fragmento florestal, pressão de caça, estrutura da vegetação e qualidade do habitat (SCHARWZKopf \& RyLANDS 1989, Peres 1997, Chiarello 1999, Cullen Jr. et al. 2000, Peres 2001, Heiduck 2002). Neste sentido, Chiarello (1999), Chiarello \& Melo (2001) e CULLEN JR. et al. (2001) realizaram censos populacionais de mamíferos diurnos em diversas áreas de Mata Atlântica, relacionando a abundância e densidade das espécies com o tamanho dos fragmentos florestais e pressão de caça. Além dos fragmentos com maior pressão de caça terem apresentado baixa densidade e biomassa de mamíferos, foi encontrado maior número de espécies em remanescentes florestais de maior área.

Particularmente com relação aos primatas, existe uma série de trabalhos realizados em fragmentos de Mata Atlântica que abordaram a riqueza, a abundância ou a densidade (Stallings et al. 1991, Pinto et al. 1993, Olmos \& Martucelli 1995, Müller 1996, Cosenza \& Melo 1998, Chiarello 1999, Chiarello \& Melo 2001, Gonzáles-Sóliz et al. 2002), mas ainda existe uma carência de dados comparativos para se entender as respostas de cada espécie à fragmentação florestal. Além disso, a maioria dos trabalhos não utiliza técnicas padronizadas de censo ou possui amostragens insuficientes, o que dificulta comparações entre as áreas (Chiarello 2003, Cullen Jr. \& Rudran 2003).

Devido ao pequeno tamanho e isolamento da maioria dos fragmentos de floresta semidecídua da Mata Atlântica, é pouco provável que populações mínimas viáveis de primatas sejam mantidas a longo prazo (Chiarello \& Melo 2001, Chiarello 2003). Portanto, essas áreas se destinam à conservação de metapopulações que deverão ser manejadas num futuro próximo para serem evitadas extinções locais de espécies (KierulfF \& RYLANDS 2003).

Existem poucos trabalhos com amostragem robusta sobre a densidade e tamanho populacional de primatas nas florestas semidecíduas paulistas (Cullen JR. et al. 2001, Martins 2004), sendo fundamental o conhecimento sobre o estado de conservação das populações de primatas existentes nestes fragmentos florestais. Assim, o objetivo deste trabalho foi estimar a abundância, densidade e tamanho populacional de três espécies de primatas em um fragmento de Mata Atlântica semidecídua no Estado de São Paulo.

\section{MATERIAL E MÉTODOS}

\section{Área de estudo}

A Fazenda São José é uma propriedade privada situada no Estado de São Paulo, entre os municípios de Rio Claro e Araras, numa altitude de $630 \mathrm{~m}$. Atualmente existem três fragmentos na fazenda com 230 ha, 185 ha e 165 ha, separados entre si principalmente por monocultura de cana-de-açúcar e manchas de seringal. O censo de primatas foi realizado no fragmento maior (230 ha) denominado Mata São José ( $47^{\circ} 28^{\prime} \mathrm{W}$ e $22^{\circ} 22^{\prime} \mathrm{S}$ ).

A Mata São José é um dos poucos remanescentes de floresta estacional semidecídua (IвGE 1992) que resta no interior do Estado de São Paulo. Esta mata apresentou maior riqueza florística quando comparada a outros remanescentes do Estado de São Paulo, com cerca de 244 espécies arbustivas e arbóreas (PAgano et al. 1995). O dossel varia de 15 a $30 \mathrm{~m}$ de altura, havendo freqüente ocorrência de lianas, cipós e bambus formando densos emaranhados.

A pluviosidade total anual foi de $1537,7 \mathrm{~mm}$ no ano de 1999 e $1601,7 \mathrm{~mm}$ no ano de 2000 , segundo os dados fornecidos pelo Centro de Análise e Planejamento Ambiental (UNESP, campus Rio Claro, São Paulo).

BRIANI et al. (2001) registraram sete espécies de roedores (Sciuridae e Muridae), três espécies de marsupiais (Didelphidae), duas de edentados (Dasypodidae) e uma espécie de tapeti (Leporidae) na Mata São José. Também relataram a presença de três espécies de primatas: macaco-prego Cebus nigritus (Goldfuss, 1809) (Cebidae), sagüi-da-serra-escuro Callithrix aurita (É. Geoffroy, 1812) (Callitrichidae) e sauá Callicebus nigrifrons (Spix, 1823) (Pitheciidae). Callicebus nigrifrons e Callithrix aurita estão inclusos na lista nacional das espécies da fauna brasileira ameaçadas de extinção no Brasil (divulgada pelo Ministério do Meio Ambiente em 22/05/2003), classificados na categoria de ameaça "vulnerável".

\section{Métodos}

Para avaliar a abundância (avistamentos/10km) e densidade (indivíduos/ $\mathrm{km}^{2}$ ) dos primatas da Mata São José foram realizados censos utilizando o método de transecções lineares (Burnham et al. 1980, Peres 1999, Buckland et al. 2001). Três trilhas paralelas e lineares de $0,95 \mathrm{~km}, 1,3 \mathrm{~km}$ e 1,65 km de comprimento foram percorridas no fragmento, a uma velocidade constante de aproximadamente $1 \mathrm{~km} / \mathrm{h}$, no período entre 6:00h e 11:30h, horários de maior atividade dos animais diurnos (Peres 1999). As trilhas distavam cerca de $500 \mathrm{~m}$ uma da outra e nenhuma trilha foi amostrada mais de uma vez no mesmo dia.

A cada avistamento foi anotado o nome da espécie, número de indivíduos e distância perpendicular entre o animal e a trilha. Esta distância foi medida diretamente com auxílio de trena métrica e, no caso de haver mais de dois indivíduos avistados num mesmo grupo, foi medida a distância perpendicular do centro do bando à trilha (Chiarello 1999, Cullen Jr. et al. 2000). 
Ao adotar este método, o observador tem que respeitar premissas tais como detectar todos os animais que estiverem na transecção, não contar o mesmo animal mais de uma vez em uma mesma transecção, medir as distâncias perpendiculares acuradamente e detectar o animal em sua posição de origem, pois os animais não devem se mover a longas distâncias antes de serem detectados pelo observador (Peres 1999, Тномas et al. 2002, Cullen JR \& Rudran 2003).

O software Distance 4.1 foi utilizado para o cálculo de densidade dos primatas (modelo "Half-normal", função cosseno, sequencial, critério de informação de Akaike) (BurNHAM et al. 1980, Тномаs et al. 1998). A densidade foi calculada para cada espécie através da fórmula $\mathrm{D}=\mathrm{N} /\left(2^{*} \mathrm{LE}{ }^{\star} \mathrm{L}\right)$, onde $\mathrm{D}=$ densidade (indivíduos $/ \mathrm{km}^{2}$ ); $\mathrm{N}=$ número total de avistamentos; $\mathrm{LE}=$ largura efetiva da área amostrada (em $\mathrm{Km}$ ), calculada pelo programa Distance; $\mathrm{L}$ = quilometragem total percorrida.

Como algumas espécies foram avistadas poucas vezes (Tab. I), os dados de distâncias perpendiculares dos três primatas foram combinados, a fim de se calcular um valor geral da largura efetiva da área amostrada e assim, uma densidade com baixo coeficiente de variação (menor que $20 \%$, como recomendado por Buckland et al. 1993). Antes de se adotar este procedimento, foi verificado que não houve diferença estatística entre os valores das distâncias perpendiculares coletados para cada espécie de primata, através de uma Análise de Variância (ANOVA de uma via, $\mathrm{p}=0$,59) (Zar 1984, SoKAl \& RohlF 1995).

O tamanho populacional de cada espécie foi obtido multiplicando-se a densidade pelo tamanho do fragmento amostrado. A biomassa de cada espécie foi obtida multiplicando-se a densidade pela massa corpórea (em quilos) obtida em FONSECA et al. (1996).

\section{RESULTADOS}

Durante 19 meses (entre julho de 1999 a janeiro de 2001) foram percorridos $125 \mathrm{~km}$ de transecções. Foram confirmadas três espécies de primatas na Mata São José, durante os censos: macaco-prego Cebus nigritus, sagüi-da-serra-escuro Callithrix aurita e sauá Callicebus nigrifrons.

A largura efetiva da área amostrada, calculada através dos valores de distâncias perpendiculares das três espécies de primatas, foi de $8,12 \mathrm{~m}$ (variando de $6,2 \mathrm{~m}$ a $10,5 \mathrm{~m}$ ).

O primata mais abundante foi Cebus nigritus (3,92 grupos/ 10km) apresentando uma densidade de 24,5 indivíduos/ $\mathrm{km}^{2}$ (Tab. I). A abundância de Callicebus nigrifrons e de Callithrix aurita foi estimada em 0,56 grupo/10km, refletindo uma densidade de 3,5 indivíduos/ $\mathrm{km}^{2}$ (Tab. I). A média do tamanho do grupo de Cebus nigritus foi de 3,79 indivíduos ( $\mathrm{cv}=9,8 \%$ ). Durante o período de estudo esse primata foi também avistado no entorno da Mata São José, forrageando nas plantações de cana-de-açúcar e milho que circundam a floresta.

Callicebus nigrifrons foi detectado freqüentemente aos pares ou solitário, tendo sido registrados três indivíduos apenas uma vez. A média do tamanho de grupo do sauá foi de
1,71 indivíduos/grupo ( $\mathrm{cv}=16,6 \%)$. Para Callithrix aurita foi obtida uma média de 3,14 indivíduos/grupo (cv = 14,6\%).

A população de macaco-prego na Mata São José foi estimada em 56 indivíduos (variando de 45 a 75 indivíduos), e a população total de sauá e sagüi-da-serra-escuro foi de 8 indivíduos (variando de 6 a 10 indivíduos) (Tab. I). Cebus nigritus apresentou a maior biomassa de primatas no fragmento, com $61,2 \mathrm{~kg} / \mathrm{km}^{2}$, enquanto Callicebus nigrifrons apresentou uma biomassa de $4,72 \mathrm{~kg} / \mathrm{km}^{2}$ e Callithrix aurita apresentou 1,01 $\mathrm{kg} / \mathrm{km}^{2}$ (Tab. I).

\section{DISCUSSÃO}

A Mata São José ainda abriga uma considerável riqueza de espécies de primatas da Mata Atlântica semidecídua. Das três espécies que ocorrem na área, duas são consideradas como vulneráveis à extinção, segundo a lista nacional das espécies da fauna brasileira ameaçada de extinção no Brasil (divulgada pelo Ministério do Meio Ambiente em 22/05/2003).

O local com maior riqueza de primatas nas matas semidecíduas é a região da Fazenda Barreiro Rico, em Anhembi (São Paulo), que inclui o bugio Alouatta guariba Cabrera, 1940 (Atelidae) e o mono-carvoeiro Brachyteles arachnoides É. Geoffroy, 1806 (Atelidae), além das três espécies avistadas na Mata São José (Galetti 1996, Martins 2004).

Não foi registrada a presença de bugio na Mata São José e o local mais próximo onde esta espécie pode ser encontrada é na Mata Santa Genebra, em Campinas (São Paulo), à cerca de $80 \mathrm{~km}$ da área de estudo (Chiarello \& GaletTi 1994).

Somente o macaco-prego foi encontrado em alta densidade na Mata São José, enquanto para o sauá e o sagüi-daserra-escuro foi registrada uma baixa densidade populacional. A densidade de macaco-prego na Mata São José foi um pouco maior em relação às densidades registradas em outros fragmentos de floresta semidecídua em São Paulo (Cullen Jr. et al. 2001) e semelhante à Reserva Florestal de Linhares no Espírito Santo (Chiarello \& Melo 2001).

A dieta generalista de Cebus nigritus pode ser um dos fatores responsáveis pela alta densidade populacional desta espécie em fragmentos florestais de pequeno porte, como a Mata São José (Spironello 1991, Galetti \& Pedroni 1994). Durante o período de estudo, esse primata foi avistado no entorno consumindo cana-de-açúcar e milho, fato observado também em outros fragmentos florestais cercados por plantações (Brown et al. 1984, Brown \& Zunino 1990, Galetti \& Pedroni 1994).

Callithrix aurita é endêmico do sudeste do Brasil e naturalmente raro (Olmos \& Martucelli 1995, Brandão \& Develey 1998). Essa espécie é predominantemente insetívora, sendo menos gomívora que as outras espécies do mesmo gênero (Muskin 1984, Ferrari et al. 1996). A persistência deste sagüi em fragmentos florestais de pequena área deve-se à grande disponibilidade de insetos que proliferam nos emaranhados de cipós e lianas (Muskin 1984, Brandão \& Develey 1998), mas sua baixa densidade poder ser reflexo de alta pressão de predação.

Revista Brasileira de Zoologia 21 (4): 827-832, dezembro 2004 
Tabela I. Informações sobre os parâmetros populacionais das três espécies de primatas avistadas na Mata São José, Rio Claro, São Paulo: número de avistamento, abundância, densidade populacional, massa corpórea, tamanho da população e biomassa (os intervalos de confiança são encontrados entre parênteses).

\begin{tabular}{lcccccc}
\hline \multicolumn{1}{c}{ Espécie } & $\begin{array}{c}\text { Massa } \\
\text { corpórea }(\mathrm{kg})^{*}\end{array}$ & $\begin{array}{c}\text { Número de } \\
\text { avistamento }\end{array}$ & $\begin{array}{c}\text { Abundância } \\
\text { (grupos/10 km) }\end{array}$ & $\begin{array}{c}\text { Densidade } \\
\text { (indivíduos/km²) }\end{array}$ & $\begin{array}{c}\text { Tamanho } \\
\text { populacional }\end{array}$ & Biomassa $\left(\mathrm{kg} / \mathrm{km}^{2}\right)$ \\
\hline Cebus nigritus & 2,50 & 49 & 3,92 & $24,5(19,6-32,6)$ & $56(45-75)$ & $61,2(49-81,5)$ \\
Callicebus nigrifrons & 1,35 & 7 & 0,56 & $3,5(2,8-4,6)$ & $8(6-10)$ & $4,72(3,78-6,21)$ \\
Callithrix aurita & 0,29 & 7 & 0,56 & $3,5(2,8-4,6)$ & $8(6-10)$ & $1,01(0,81-1,33)$ \\
\hline
\end{tabular}

* Segundo FonseCA et al. (1996).

A densidade populacional de calitriquídeos foi registrada em alguns fragmentos florestais do Espírito Santo (CHIARELLO \& Melo 2001) e São Paulo (Martins 2004). Em um fragmento florestal de tamanho similar à da Mata São José (Putiri, ES, com 210 ha), a densidade de Callithrix geoffroyi É Geoffroy, 1812 (Callitrichidae) foi semelhante à densidade de C. aurita na Mata São José. Nos demais fragmentos capixabas, a menor densidade registrada foi cerca de cinco vezes maior que a encontrada na Mata São José. Em fragmentos de floresta semidecídua paulista maiores que a Mata São José, situados na Fazenda Barreiro Rico (Anhembi), a abundância de Callithrix aurita foi menor que na área estudada (MARTINS 2004).

Chiarello (2000) sugeriu que a baixa abundância de Callithrix nos pequenos fragmentos florestais deve-se à alta predação por felinos de menor porte como o gato-maracajá Leopardus wiedi Schinz, 1821. Na Mata São José, a jaguatirica Leopardus pardalis Linnaeus, 1758 pode predar C. aurita, uma vez que utiliza eventualmente a área estudada como um "trampolim ecológico", em busca de abrigo e fonte de alimento em meio às plantações (Chiarello 2000, Cullen Jr. et al. 2000).

Comparando a densidade de Callicebus Thomas, 1903 (Pitheciidae) em diversos fragmentos florestais (PINTo et al. 1993, Müller 1996, Cosenza \& Melo 1998, Chiarello 1999, Chiarello \& Melo 2001), a mata estudada apresentou a menor densidade registrada. No complexo de florestas de Ituberá (BA) foram registrados indivíduos de Callicebus nigrifrons em fragmentos de 100 a 300 ha, nas bordas da mata e em fragmentos estreitos e isolados por plantação (FLESHER 1999). Diversos estudos relataram que essa espécie vive em florestas secundárias e raramente em florestas primárias, bem como registrada em ambientes com distribuição em manchas de floresta (STALLINGS \& Robinson 1991, Fonseca et al. 1994, Rylands et al.1997).

É possível que esses três primatas utilizem algumas áreas de mata dos dois fragmentos vizinhos à Mata São José ou a mata ciliar do Ribeirão Claro, apesar de parte da vegetação ter sido queimada em vários incêndios decorrentes da queima de cana-de-açúcar. Em 2001 o fogo afetou principalmente a parte sudoeste da Mata São José, reduzindo ainda mais o habitat e disponibilidade de alimento para as espécies locais. Na Floresta Nacional de Rio Preto (Espírito Santo), Callicebus nigrifrons só foi encontrado em áreas de floresta onde incêndios não ocorreram (Chiarello \& Melo 2001).
O pequeno tamanho populacional das espécies de primatas na Mata São José (máximo de 75 indivíduos de macaco - prego e máximo de 10 indivíduos de sauás e de sagüis-daserra-escuro) certamente não garante a sobrevivência destas espécies no fragmento florestal a longo prazo. Um estudo realizado com cerca de 100 espécies de vertebrados sugeriu que é necessário uma população mínima de cerca de 7.000 indivíduos para que esta seja viável e não sofra com os efeitos demográficos, genéticos e estocásticos (REED et al. 2003).

Chiarello (2000) sugeriu que somente fragmentos florestais maiores que 20.000 ha são capazes de manter populações viáveis de primatas a longo prazo. Baseado no tamanho populacional médio obtido na Mata São José, seriam necessários cerca de 14.000 ha de floresta semidecídua para ser mantida uma população de no mínimo 500 indivíduos de $C$. aurita e $C$. nigrifrons. No Estado de São Paulo, o único fragmento de floresta semidecídua de grande porte é o Parque Estadual Morro do Diabo, com cerca de 35.000 ha. O segundo maior fragmento de Mata Atlântica semidecídua possui apenas 2.200 ha (Estação Ecológica de Caetetus) (Cullen JR. et al. 2000). Isto demonstra que a grande maioria dos fragmentos florestais paulistas de Mata Atlântica semidecídua não possui tamanho de área suficiente para manter populações viáveis de primatas.

A implantação de corredores ecológicos, conectando a Mata São José a outros fragmentos florestais próximos, acrescentaria uma área de floresta de no máximo 500 ha à paisagem dominada por plantações e pastagens. Assim, a translocação dos primatas ameaçados e com baixas densidades populacionais da Mata São José é a medida de manejo mais plausível, que pode evitar a extinção local destas populações.

\section{AGRADECIMENTOS}

Ao CNPq pela bolsa PIBIC concedida à C.S.S. Bernardo, aos funcionários do Departamento de Ecologia do Instituto de Biociências da UNESP-Rio Claro, à FAPESP (96/10464-4 e 01/ 14463-5) pelo apoio financeiro e à ONG Idea Wild pelos equipamentos doados. Agradecemos também aos consultores Alfredo Ricardo Langguth Bonino, Fernando Dias de Avila Pires e a um consultor anônimo pelos valiosos comentários. Gabriele M. Preiskorn, Mauricio N. Godoy e Paulo Rubim auxiliaram na coleta de dados na Mata São José e Ricardo S. 
Bovendorp auxiliou nas análises de estimativa de densidade. M.Galetti recebe bolsa de produtividade em pesquisa do CNPq.

\section{REFERÊNCIAS BIBLIOGRÁFICAS}

Bierregaard, R.O. JR.; T.E. Lovejoy; V. Kapos; A.A. dos Santos \& R.W. Hutchings. 1992. The biological dynamics of tropical rainforest fragments. Bioscience, Washington, 42: 859-866.

Brandẽo, L.D. \& P.F. Develey. 1998. Distribution and conservation of the buffy tufted-ear marmoset, Callithrix aurita, in lowland coastal atlantic forest, southeast Brazil. Neotropical Primates, Belo Horizonte, 6 (3): 86-88.

Briani, D.C.; R.T. Santori; M.V. Vieira \& N. Gobbi. 2001. Mamíferos não-voadores de um fragmento de mata mesófila semidecídua do interior do Estado de São Paulo, Brasil. Holos Environment, Rio Claro, 1 (2): 141-149.

Brown, A.; S. Chalukian \& L. Malmierca. 1984. Habitat y alimentacion de Cebus nigritus en N.O. argentino y la disponibilidad de frutos en al dossel arboreo. Revista del Museo Argentino Ciências Naturais Bernardino Rivadavia, Buenos Aires, 13: 273-280.

Brown, A.D. \& G.E. Zunino. 1990. Dietary variability in Cebus nigritus in extreme habitats: evidence for adaptability. Folia Primatologica, Switzerland, 54: 187-195.

Buckland, S.T.; D.R. Anderson; K.P. Burnham; J.L. LAaKe. 1993. Distance sampling. Estimating abundance of biological populations. London, Chapmann \& Hall, 432p.

Buckland, S.T.; D.R. Anderson; K.P. Burnham; J.L. LaAke; D.L. Borchers \& L. Thomas. 2001. Introduction to distance sampling. Oxford, Oxford University Press, 568p.

Burnham, K.P.; D.R. ANDERson \& J.L. LAAKE. 1980. Estimation of density from line transect sampling of biological populations. Wildlife Monographics, Oxford, 72: 1-202.

Chiarello, A.G. 1999. Effects of fragmentation of the Atlantic forest on mammal communities in southeastern Brazil. Biological Conservation, Liverpool, 89: 71-82.

. 2000.Conservation value of a native forest fragment in a region of extensive agriculture. Revista Brasileira de Biologia, São Carlos, 60 (2): 237-247.

Chiarello, A.G. \& M. Galetti. 1994. Conservation of the brown howler monkey in southeast Brazil. Oryx, Cambridge, 28: 37-42.

Chiarello, A.G. \& F.R. de Melo. 2001. Primate population densities and sizes in Atlantic forest remnants of northern Espirito Santo, Brazil. International Journal of Primatology, Londres, 22 (3): 379-396.

Chiarello, A.G. 2003. Primates of brazilian Atlantic forest: the influence of forest fragmentation on survival, p. 99-118. In: L.K. Marsh (Ed.). Primates in fragments: ecology and conservation. New York, Kluwer Academic \& Plenum Publishers, 428p.

Cosenza, B.A.P. \& F.R. Melo. 1998. Primates of the Serra do Brigadeiro State Park, Minas Gerais, Brazil. Neotropical
Primates, Belo Horizonte, 6 (1): 18-20.

Cullen JR., L. \& R. Rudran. 2003. Transectos lineares na estimativa de densidade de mamíferos e aves de médio e grande porte, p.169-179. In: L. Cullen Júnior; R Rudran; C. ValladaRES-PADUA (Eds). Métodos de estudos em biologia da conservação e manejo da vida silvestre. Curitiba, Editora UFPR e Fundação o Boticário de Proteção à Natureza, 667p.

Cullen JR., L.; R.E. Bodmer \& C.V. Padua. 2000. Effects of hunting in habitat fragments of the Atlantic forest, Brazil. Biological Conservation, Liverpool, 95: 49-56.

2001. Ecological consequences of hunting in Atlantic forest patches, São Paulo, Brazil. Oryx, Cambridge, 35: 137-144.

DEAN, W. 1996. A ferro e fogo: a história e a devastação da Mata Atlântica brasileira. São Paulo, Companhia das Letras, 504p.

Ferrari, S.; K.M. CorrêA \& P.E.G. Coutinho. 1996. Ecology of the "southern" marmosets (Calithrix aurita and Callithrix flaviceps): How different, how similar?, p.157-171. In: M.A. NoRConk; A.L. Rosenberger; P.A. Garber (Eds). Adaptative radiations of Neotropical Primates. Illinois, Plenum Press, 490p.

Flesher, K. 1999. Primates of Ituberá Forest Complex, Brazil. Neotropical Primates, Belo Horizonte, 7 (4): 127-131.

Fonseca, G.A.B.; A.B. Rylands; C.M.R. Costa; R.B. Machado \& Y.L.R. LeIte. 1994. Livro Vermelho dos Mamíferos Brasileros ameaçados de Extinção. Belo Horizonte, Fundação Biodiversitas. 459p.

Fonseca, G.A.B.; G. Hermmann; Y.L.R. Leite; R.A. Mittermeier; A.B. Rylands \& J.L. Patton. 1996. Lista Anotada dos Mamíferos do Brasil. Occasional Papers in Conservation Biology, Chicago, 4: 1-38.

Galetti, M. 1996. Comportamentos antipredatórios de quatro espécies de primatas no sudeste do Brasil. Revista Brasileira de Biologia, São Carlos 56: 203-209.

Galetti, M. \& F. Pedroni. 1994. Seasonal diet of capuchin monkey (Cebus nigritus) in a semideciduous forest in southeast Brazil. Journal of Tropical Ecology, Cambridge, 10: 27-39.

GonzÁlez-Sólis,J.; J.C. Guix; E. Mateos J.C. \& L. Llorens. 2002. Density estimates, group size and habitat use of monkeys (mammals: cebidae), p. 111-125. In: E. Mateos J.C.; A.S. Guix $\&$ K. PisciotTA (Eds). Censuses of vertebrates in a Brazilian Atlantic rainforest area: the Paranapiacaba fragment. Barcelona, Universidad de Barcelona, 217p.

HeIDUCK, S. 2002. The use of disturbed and undisturbed forest by masked-titi monkeys Callicebus personatus melanochir is proportional to food availability. Oryx, Cambridge, 36 (2): 133-139.

IbGe. 1992. Manual técnico da vegetação brasileira. Rio de Janeiro, Fundação Instituto Brasileiro de Geografia e Estatística, Série Manuais Técnicos em Geociências, 92p.

KierulfF, M.C.M. \& A.B. Rylands. 2003. Census and distribution of the golden lion tamarin (Leontopithecus rosalia). American Journal of Primatology, Washington, 59 (1): 29-44. 
Martins, M.M. 2004. Density of primates in four semi-deciduous forest fragments of São Paulo, Brazil. Biodiversity and Conservation, New York, 23: 1-9.

Müller, K.H. 1996. Ranging in masked titi monkeys (Callicebus nigrifrons) in Brazil. Folia Primatologica, Switzerland, 65: 224-228.

Muskin, A. 1984. Field notes and geographic distribution of Callithrix aurita in eastern Brazil. American Journal of Primatology, Washington, 7: 377-380.

Olmos,F. \& P. Martuscelli. 1995. Habitat and distribution of the buffy-tufted-ear marmoset Callithrix aurita in São Paulo State, Brazil, with notes on its natural history. Neotropical Primates, Belo Horizonte, 3 (3): 75-79.

Pagano, S.N.; H.F. Leitão Filho \& O. Cavassan. 1995. Variação temporal da composição florística e estrutura fitossociológica de uma floresta mesófila semidescídua - Rio Claro Estado de São Paulo. Revista Brasileira de Biologia, Rio de Janeiro, 55 (2): 241-258.

PERES, C.A. 1997. Effects of habitat quality and hunting pressure on arboreal folivore densities in neotropical forests: A case study of howler monkeys (Alouatta spp.). Folia Primatologica, Switzerland, 68: 199-222.

-1999. General guidelines for standardizing linetransect surveys of tropical forest primates. Neotropical Primates, Belo Horizonte, 7 (1): 11-16.

. 2001. Synergistic effects of subsistence hunting and habitat fragmentation on Amazonian forest vertebrates. Conservation biology, Florida, 15 (6): 1490-1505.

Pinto, L.P.; C.M.R. Costa; K.B. Strier \& G.A.B. Fonseca. 1993. Habitat density and group size of primates in a Brazilian tropical forest. Folia primatologica, Switzerland, 61: 135-143.

Reed, D.H.; J.J. O'Grady; B.W. Brook; J.D. Ballou \& R. Frankham. 2003. Estimates of minimum viable population sizes for vertebrates and factors influencing those estimates. Biological conservation, Liverpool, 113: 23-34.

Rylands, A.B.; R.A. Mittermeier \& E. Rodriguez-Luna. 1997. Conservation of neotropical primates: threatened species and an analysis of primate diversity by country and region. Folia primatologica, Switzerland, 68: 134-160.

SchwarzKopf, L. \& A.B. Rylands. 1989. Primates species richness in relation to habitat structure in Amazonian rainforest fragments. Biological conservation, Liverpool, 48: 1-12.

SmA (Secretaria do Meio Ambiente). 2000. Atlas das unidades de conservação ambiental do Estado de São Paulo. São Paulo, Governo do Estado de São Paulo, 38p.

Sokal, R.R. \& F.J. Rohlf. 1995. Biometry. New York, W.H. Freeman, $3^{\text {rd }}$ ed., $887 \mathrm{p}$.

Sos Mata AtLÂNTICA \& InPE. 1998. Evolução dos remanescentes florestais e ecossistemas associados do domínio de Mata Atlântica. São Paulo, SOS Mata Atlântica e Instituto de Pesquisas Espaciais, 43p.

SPIRONElo, W.R. 1991. Importância dos frutos de palmeiras (Palmae) na dieta de um grupo de Cebus apella (Cebidae, Primates) na Amazônia Central, p.285-296. In: AB. Rylands \& A.T. Bernardes (Eds). A primatologia no Brasil 3. Brasília, SBPr, 420p.

Stallings, J.R. \& J.G. Robinson. 1991. Disturbance, forest heterogeneity and primate communities in a Brazilian Atlantic Forest Park, p. 357-358. In: AB. Rylands \& A.T. Bernardes (Eds). A Primatologia no Brasil 3. Brasília, SBPr, 420p.

Thomas, L.; J.L. Laake; J.F. Derry; S.T. BuckLand; D.L. Borchers; D.R. Anderson; K.P. Burnham; S. Strindberg; S.L. Hedley; M.L. Burt; F.F.C. Marques; J.H. Pollard \& R.M. Fewster. 1998. Distance 3.5. Research unit for wildlife population assesment, Reino Unido, University of St. Andrews. http:// www.ruwpa.st-and.ac.uk/distance/.

ThOMAs, L.; S.T. BucKLAND; K.P. BuRnham; D.R. ANDERSON; J.L. LAAKE; D.L. Borchers \& S. Strindberg. 2002. Distance sampling. Encyclopedia of environmetrics, Chichester, 1: 544-552.

Turner, I.M. \& R.T. CorletT. 1996. The conservation value of small, isolated fragments of lowland tropical rainforest. Trends in ecology and evolution, Oxford, 11: 663-668.

ZaR, J.H. 1984. Biostatistical Analysis. Englewood Cliffs, Prentice-Hall, $2^{\text {nd }}$ ed., 718p.

Recebido em 02.II.2004; aceito em 22.X.2004. 